\title{
Application of a radiation pneumonitis prediction model in patients with locally advanced lung squamous cell cancer
}

\author{
Ailu Wu ${ }^{1,2 \#}$, Ziyang Zhou ${ }^{1,3 \#}$, Yipeng Song ${ }^{1,2 \#}$, Shixiong Liang ${ }^{1,4}$, Fangjuan Li $^{1}$ \\ ${ }^{1}$ Department of Radiation Oncology, Shanghai Pulmonary Hospital, Tongji University School of Medicine, Shanghai, China; ${ }^{2}$ Department of \\ Radiation Oncology, The Affiliated Yantai Yuhuangding Hospital, Qingdao University School of Medicine, Yantai, China; ${ }^{3}$ Department of Radiation \\ Oncology, The First Affiliated Hospital, Zhejiang University School of Medicine, Hangzhou, China; ${ }^{4}$ Department of Radiation Oncology, Guangxi \\ Medical University Cancer Hospital, Nanning, China \\ Contributions: (I) Conception and design: All authors; (II) Administrative support: All authors; (III) Provision of study materials or patients: All \\ authors; (IV) Collection and assembly of data: All authors; (V) Data analysis and interpretation: S Liang, F Li; (VI) Manuscript writing: All authors; \\ (VII) Final approval of manuscript: All authors. \\ "These authors contributed equally to this work. \\ Correspondence to: Fangjuan Li. Department of Radiation Oncology, Shanghai Pulmonary Hospital, Tongji University School of Medicine, Zhengmin \\ Road, Yangpu District, Shanghai 200433, China. Email: lifangjuan12345@sina.com; Shixiong Liang. Guangxi Medical University Cancer Hospital, \\ Hedi Road, Qingxiu District, Nanning 530021, China. Email: shixliang12345@sina.com.
}

Background: The aim of the present study was to establish a new prediction model for radiation pneumonitis (RP) in locally advanced non-small cell lung cancer (LA-NSCLC) patients before and after radiotherapy.

Methods: The study involved 153 patients. Age, arterial partial oxygen pressure $\left(\mathrm{PO}_{2}\right)$, forced vital capacity, pulmonary emphysema (PE), subclinical interstitial lung disease (sILD), and dosimetric parameters, such as mean lung dose and percentage of lung volume, and a dose >5/20 Gy (V5/V20), were considered candidate RP predictors.

Results: Of the 153 eligible patients, 33 (21.6\%) developed RP, 68 had PE (43.8\%), and 24 (15.7\%) had sILD. Grades 2, 3, and 5 RP were scored in 17 (11.1\%), 15 (9.8\%), and $1(0.7 \%)$ patient/s, Grade 4 RP was not observed. Grades 1, 2, and 3 PE were scored in 45 (29.4\%), 22 (14.4\%), and 1 (0.7\%) patient/s. Grades 0 and 1 sILD were observed in 129 (84.3\%) and 24 (15.7\%) patients. Univariate analysis found age, PE, and sILD to be significantly correlated with grade $\geq 2$ RP. Multivariate analysis revealed age $>68$ years, PE grade $>1$, and sILD grade $\geq 1$ as independent risk factor for grade $\geq 2$ RP in LA-NSCLC with squamous cell carcinoma (SCC). Finally, a new predictive risk score (PRS) comprised of these factors was developed. The PRS score was $0,3-5$, and 6-11 when the cumulative incidence of grade $\geq 2 \mathrm{RP}$ was $8.8 \%$ (5 patients), $13 \%$ (3 patients), and $84.6 \%$ (13 patients) $(\mathrm{P}=<0.001)$.

Conclusions: Age, PE, and sILD could independently and significantly predict RP in LA-NSCLC with SCC.

Keywords: Locally advanced non-small cell lung cancer; radiation pneumonitis; age; pulmonary emphysema; subclinical interstitial lung disease

Submitted Jan 20, 2021. Accepted for publication Apr 17, 2021.

doi: $10.21037 / \mathrm{apm}-21-459$

View this article at: http://dx.doi.org/10.21037/apm-21-459 


\section{Introduction}

Global lung cancer incidence and mortality remain high, although they can be effectively reduced by tobacco smoking cessation (1). Non-small cell lung cancer (NSCLC) is the most prevalent lung cancer type, making up $85 \%$ of lung cancer cases (2). Squamous cell carcinoma (SCC) and adenocarcinoma (ADE) are the main histological subtypes of NSCLC. Locally advanced (LA)-NSCLC refers to clinical stage IIIA or IIIB NSCLC, and is mainly treated using concurrent chemoradiotherapy (CCRT) (3); however, this strategy is limited by radiation pneumonitis (RP), which is an inevitable dose-limiting adverse effect of LA-NSCLC radiotherapy. Therefore, to treat LA-NSCLC effectively and safely, an accurate method of predicting RP is needed.

Radiation pneumonitis (RP) is a part of radiationinduced lung injury. Its injury and repair is a complex pathophysiological process involving and interacting with many cells and cytokines. Early diagnosis and treatment can reduce lung injury and protect lung function. Past studies have correlated RP risk with various patients' characteristics, including age, sex, smoking history, genetic predisposition, tumor location, low Karnofsky performance status (KPS), and pre-existing lung disease $(4,5)$. Dosimetric parameters have also been associated with RP, especially mean lung dose (MLD) and V20, which are widely accepted as RP risk factors. However, despite a strong association with RP, these findings have been inconsistent due to small sample sizes and different statistical methods that limit the discriminative ability of such risk factors (6-8). Various parameters have been tentatively combined to predict RP, but their predictive value is often lost (9).

We previously reported pulmonary emphysema (PE), a chronic obstructive pulmonary disease (COPD) subtype, as a risk factor in patients with LA-NSCLC after radiotherapy, especially those with SCC (10). Pre-existing radiological interstitial lung abnormalities (ILAs) are clinical risk factors for severe RP in patients with small cell lung cancer (11). $\mathrm{RP}$ tends to be more common in patients with subclinical interstitial lung disease (sILD), which includes undiagnosed patients who have ILA, as revealed by chest computed tomography (CT) scans $(12,13)$. Current RP models do not adequately consider sILD and PE in patients treated with conventionally fractionated radiotherapy. In the present study, we extend our earlier data by combining it with additional patient characteristics and dosimetric parameters in a new model for predicting RP in LA-NSCLC. In the present retrospective study, age, PE, and sILD at baseline CT were independently and significantly associated with RP incidence in LA-NSCLC patients with SCC. We combine these three factors to establish a prediction model which is more effective and simpler than the single-factor model. We present the following article in accordance with the STARD reporting checklist (available at http://dx.doi.org/10.21037/ apm-21-459).

\section{Methods}

\section{Patients}

The present study was approved by the ethical review committee of Shanghai pulmonary hospital, Tongji University School of Medicine, China. A total of 153 patients treated with radical radiotherapy at our institution from January 2014 to January 2015 were recruited into the study. The patient inclusion criteria were as follows: (I) KPS score $>70$ with no apparent pulmonary atelectasis; (II) could endure the total dose of 58-66 Gy with common fractionation (1.8-2.0 Gy/fraction/day); (III) received radiotherapy concurrently with chemotherapy; (IV) were followed up for $<6$ months without apparent local-regional recurrence; and $(\mathrm{V})$ had lung imaging features evaluated before and after radiotherapy. Observation indicators included general patient information (sex, age, smoking history, tumor location), lung function test indicators (forced expiratory volume in $1 \mathrm{~s}$, forced vital capacity, blood gas analysis), CT image examination (PE, sILD), and DVH (Dose volume histogram) parameters. Eligible participants had stage IIIA or IIIB NSCLC and were undergoing definitive radiotherapy without the option for surgery. All procedures performed in this study involving human participants were in accordance with the Declaration of Helsinki (as revised in 2013). Individual consent for this retrospective analysis was waived.

\section{Radiotherapy}

Patients received CT planning scans during quiet breathing while immobilized in a supine position with arms raised above their heads. The designed and normalized radiotherapy plans were based on 5 -mm thickness and 5 -mm interval CT scans obtained at the treatment location using an ADAC Pinnacle (Philips Medical Systems, Royal Philips of the Netherlands, Amsterdam, The Netherlands) treatment planning system. Treatments were delivered by linear accelerators with $6 \mathrm{MV}$ photons using 3D-conformal radiation therapy, or intensity modulated radiation therapy. Gross tumor volume (GTV) was contoured in sequential axial 


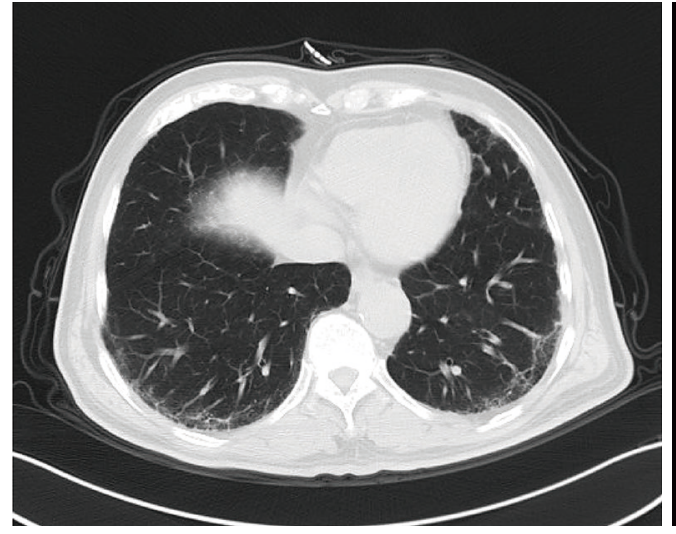

Grade 1

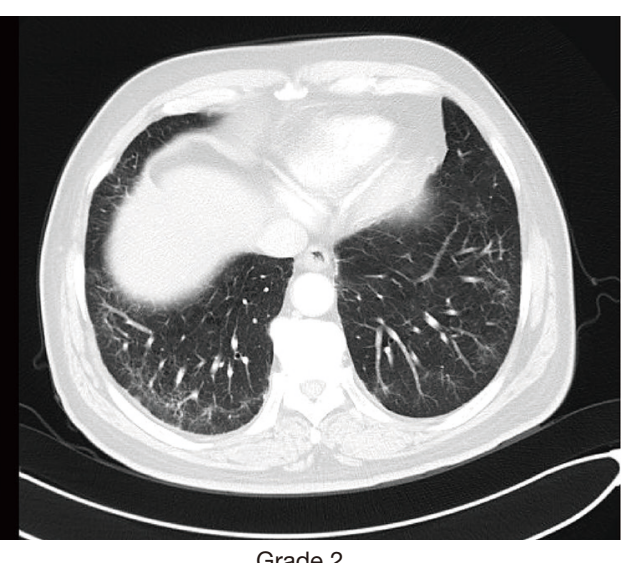

Grade 2

Figure 1 Computed tomography features of subclinical interstitial lung disease grade. Focal or unilateral ground-glass attenuation, focal or unilateral reticulation, and patchy ground-glass abnormality ( $<5 \%$ of the lung) (grade 1$)$. Non-dependent ground-glass abnormality affecting $>5 \%$ of any lung zone (grade 2 ).

CT images, and included the primary tumor and metastatic lymph node drainage area. Clinical target volume (CTV) was an increase of $0.5-1.0 \mathrm{~cm}$ on the GTV edge and included selective regional lymph nodes. Planning target volume (PTV) was generated by adding a 5 -mm axial plane margin and a $10-\mathrm{mm}$ margin, in the longitudinal direction, to the CTV. The total dose covered $95 \%$ of the PTV. MLD and volumes of the lung receiving a minimum of $5,10,20$, and 30 Gy (V5, V10, V20, and V30, respectively) were extracted based on the physical dose. The median total dose was 60 Gy (range, 58-66 Gy). The fraction dose was 1.8-2.5 Gy (median: 2.0 Gy).

\section{Chemotherapy}

The chemotherapy regimen was as described previously (10). Briefly, 22 patients received concurrent chemotherapy, while 131 patients received sequential chemotherapy.

\section{sILD and PE evaluation based on CT}

The sILD evaluation was based on high-resolution CT scans (14). CT results were evaluated by 2 chest radiologists as follows: no ILD=grade 0 , equivocal for $\mathrm{ILD}=$ grade 1 , suspicious for ILD=grade 2, and ILD=grade 3 (Figure 1). Equivocal for ILD is indicated by focal or unilateral ground-glass attenuation, focal or unilateral reticulation, and patchy ground-glass abnormality occupying $<5 \%$ of the lung zone. Suspicious for ILD refers to non-dependent ground-glass abnormality affecting $>5 \%$ of any lung zone.
ILD refers to multilobe bilateral fibrosis associated with subpleural honeycomb and traction bronchiectasis.

Before treatment, PE examination was done based on low-attenuation areas (LAAs) on CT scans. Based on Tsujino et al.'s criteria (15), PE falls into 5 grades: grade 0 (no LAAs), grade 1 (sparse, scattered, small LAAs $\leq 5 \mathrm{~mm}$ in diameter), grade 2 (adjacent LAAs $\leq 10 \mathrm{~mm}$ in diameter), grade 3 (adjacent LAAs $>10 \mathrm{~mm}$ in diameter and/or indistinguishable from each other), and grade 4 (absence of normal lung parenchyma). For specific details, please refer to our previous research reports (10).

\section{Evaluation of RP}

RP grading followed Common Terminology Criteria for Adverse Events, version 4, guidelines. Patients whose adverse events information was unavailable were excluded from the analysis. RP diagnosis and grading have been described previously. The endpoint for this analysis was grade $\geq 2 \mathrm{RP}$ or symptomatic pulmonary fibrosis.

\section{Follow-up}

Patients were followed-up weekly for up to 6 months, and after that, every 1-3 months. Follow-up was for at least 1 year. CT scans was performed at each follow-up.

\section{Statistical analysis}

Patient characteristics and dosimetric parameters were 
analyzed using Pearson's $\chi^{2}$-test or continuity correction tests. When factors were found to be significant by univariate analysis, they were subjected to multivariate analysis to develop a prediction model. Spearman's correlation test was used to evaluate relationships between RP grade and PE or ILD grade. Kaplan-Meier and receiver-operating characteristic (ROC) curve analyses were used to predict grade $\geq 2 \mathrm{RP}$. Statistical analyses were done using SPSS version 13.0 (SPSS, Chicago, IL, USA). $\mathrm{P}<0.05$ indicated statistical significance.

\section{Results}

\section{Incidence of RP, sILD, and PE}

Participant characteristics are shown in Table 1. Median patient follow-up duration was 10 months. Thirty-three (21.6\%) patients had RP; 17 (11.1\%), 15 (9.8\%), 1 (0.7\%) patient/s had grades 2, 3 and $5 \mathrm{RP}$, respectively. There were no grade $4 \mathrm{RP}$ patients. RP was detected 1-11 months after radiotherapy. Of the 153 patients, $68(43.8 \%)$ had PE. Of these, 45 (29.4\%), 22 (14.4\%), and $1(0.7 \%)$ had PE grades 1,2 , and 3, respectively. A total of $129(84.3 \%)$ patients had grade 0 sILD, while 24 (15.7\%) had grade 1 sILD. No patient had mild (grade 2) or moderate ILD (grade 3).

\section{Factors associated with RP}

The univariate analysis indicated that age, PE, and SILD were correlated with grade $\geq 2 \mathrm{RP}$ (Table 2). To assess if these factors are consistent in various pathological types, we carried out the analysis in different pathologies. The results showed that sILD was significantly correlated with grade $\geq 2 \mathrm{RP}$ for patients with $\mathrm{ADE}$. In SCC patients, age, $\mathrm{PE}$, sILD, arterial partial oxygen pressure $\left(\mathrm{PO}_{2}\right)$, and MLD exhibited a similar correlation. Results from the SCC patient analysis were similar to those of the total sample analysis. Therefore, we performed multivariate analysis in SCC patients, which showed that age $>68$ years, PE grade $>1$, and sILD grade $\geq 1$ on CT baseline were significantly correlated with grade $\geq 2$ RP (Table 3). Because beta coefficients for each significant variable could predict the incidence of grade $\geq 2 \mathrm{RP}$, we matched corresponding scores by beta coefficient. Eventually, a new model, the predictive risk score (PRS), was developed for LA-NSCLC using the following formula: PRS=5 (if subclinical ILD grade $\geq 1$ ) +3 (if age $>68$ years) +3 (if $\mathrm{PE}$ grade $>1$ ). The scoring system was based on Washko et al.'s and Satoh et al.'s studies $(16,17)$.
Table 1 Patient characteristics

\begin{tabular}{|c|c|}
\hline Demographics/characteristic & No. of patients (\%) \\
\hline \multicolumn{2}{|l|}{ Sex } \\
\hline Male & $140(91.5)$ \\
\hline Female & $13(8.5)$ \\
\hline Age (years), median [range] & 63 [40-85] \\
\hline \multicolumn{2}{|l|}{ Pathology } \\
\hline Adenocarcinoma & $28(18.3)$ \\
\hline Squamous cell carcinoma & $93(60.8)$ \\
\hline Other & $32(20.9)$ \\
\hline \multicolumn{2}{|l|}{ Stage } \\
\hline IIIA & $55(35.9)$ \\
\hline IIIB & $98(64.1)$ \\
\hline \multicolumn{2}{|l|}{ Smoking history } \\
\hline Yes & $94(61.4)$ \\
\hline No & $59(38.6)$ \\
\hline \multicolumn{2}{|l|}{ Tumor location } \\
\hline Upper lobe & 87 (56.9) \\
\hline Middle lobe & $29(18.9)$ \\
\hline Lower lobe & $37(24.2)$ \\
\hline \multicolumn{2}{|l|}{ Chemotherapy } \\
\hline Concurrent & $22(14.4)$ \\
\hline Chemotherapy first & $123(80.4)$ \\
\hline Radiotherapy first & $8(5.2)$ \\
\hline \multicolumn{2}{|l|}{ RP grade } \\
\hline $0-1$ & $120(78.4)$ \\
\hline$\geq 2$ & $33(21.6)$ \\
\hline \multicolumn{2}{|l|}{ PE grade } \\
\hline 0 & $85(55.5)$ \\
\hline 1 & $45(29.4)$ \\
\hline$\geq 2$ & $23(15.1)$ \\
\hline \multicolumn{2}{|l|}{ slLD grade } \\
\hline 0 & $129(84.3)$ \\
\hline$\geq 1$ & $24(15.7)$ \\
\hline
\end{tabular}

PE, pulmonary emphysema; RP, radiation pneumonitis; sILD, subclinical interstitial lung disease.

Next, we put this scoring system into practical application in LA-NSCLC patients with SCC. The calculated PRS of all 93 SCC patients ranged from 0 to 11 (median: 0 ). The 
Table 2 Univariate analysis for risk factors of grade $\geq 2 \mathrm{RP}$

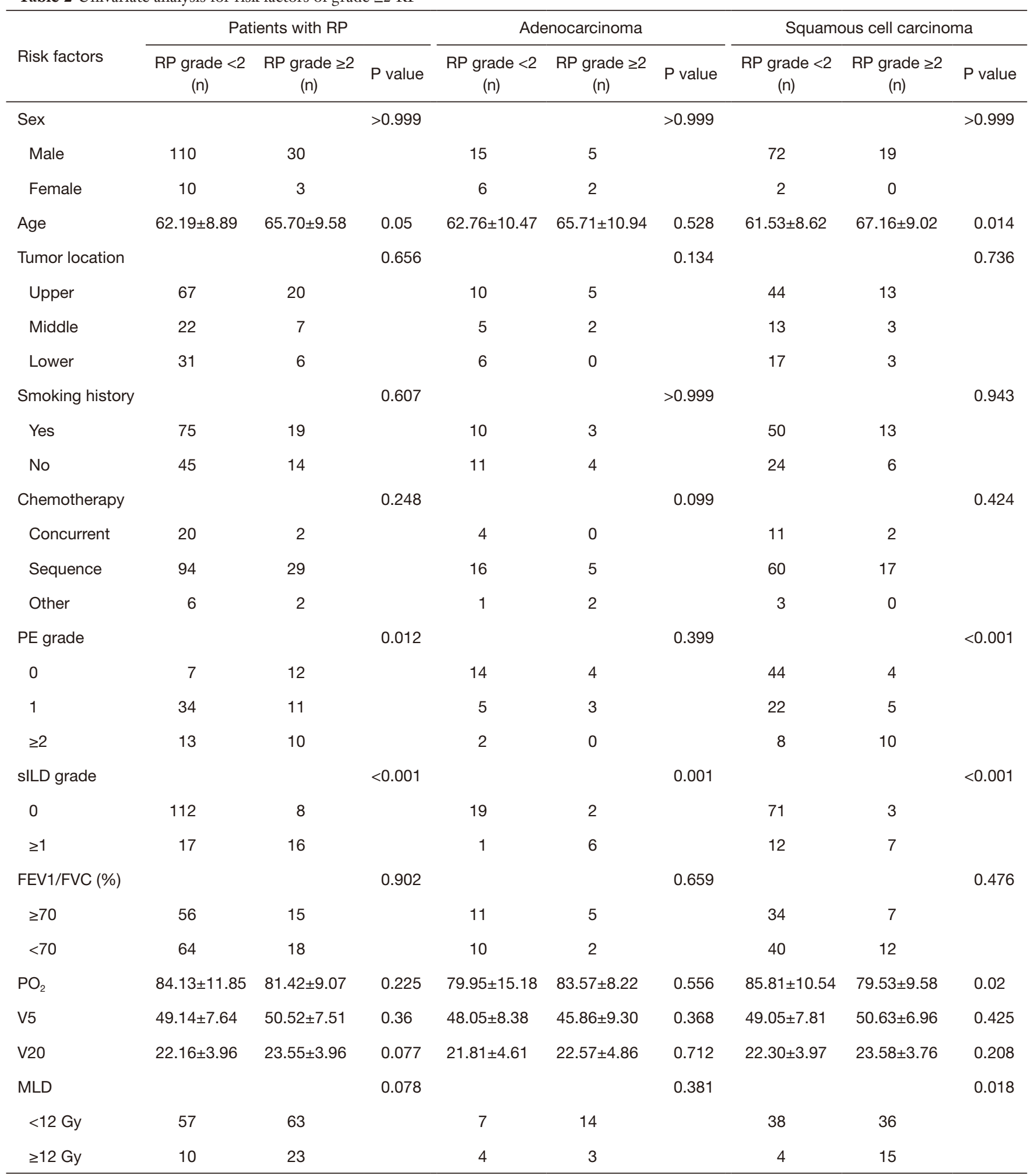

FEV1, forced expiratory volume in $1 \mathrm{~s}$; FVC, forced vital capacity; MLD, mean lung dose; PE, pulmonary emphysema; $\mathrm{PO}_{2}$, arterial partial pressure of oxygen; RP, radiation pneumonitis; sILD, subclinical interstitial lung disease; V5 or V20, percentages of the pulmonary volume irradiated exceeding 5 or 20 Gy. 
Table 3 Multivariate analysis for risk factors of grade $\geq 2 \mathrm{RP}$

\begin{tabular}{lccc}
\hline \multirow{2}{*}{ Risk factors } & & Patients with RP grade $\geq 2$ \\
\cline { 2 - 4 } & $\beta$ value & OR $(95 \%$ Cl) & P value \\
\hline Age $(\leq 68$ vs. $>68$ years) & 1.485 & $4.415(1.038-18.786)$ & 0.044 \\
PE grade (grade $\leq 1$ vs. grade $>1)$ & 1.522 & $4.581(1.078-19.473)$ & 0.039 \\
sILD grade (grade $<1$ vs. grade $\geq 1)$ & 2.577 & $13.157(2.090-82.812)$ & 0.006 \\
$\mathrm{PO}_{2}(\geq 90 \%$ vs. $<90 \%)$ & 0.377 & $1.458(0.291-7.300)$ & 0.646 \\
$\mathrm{MLD}(<12$ vs. $\geq 12$ Gy) & 0.987 & $2.684(0.621-11.587)$ & 0.186 \\
\hline
\end{tabular}

$\mathrm{Cl}$, confidence interval MLD, mean lung dose; OR, odds ratio; $\mathrm{PE}$, pulmonary emphysema; $\mathrm{PO}_{2}$, arterial partial pressure of oxygen; RP, radiation pneumonitis; sILD, subclinical interstitial lung disease.

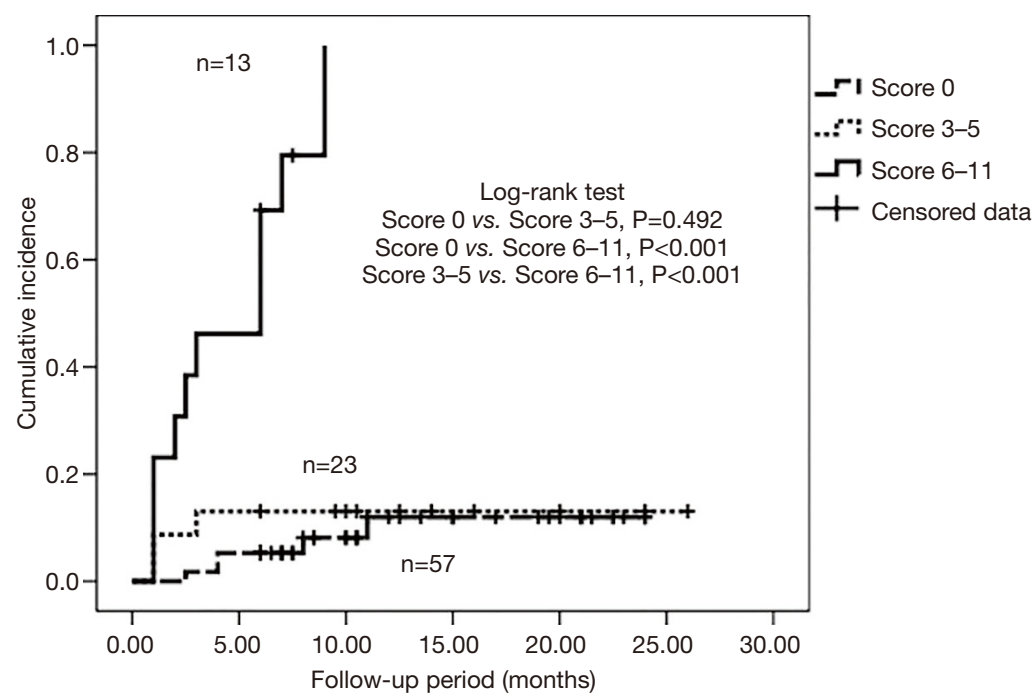

Figure 2 Cumulative incidence curves of grade $\geq 2$ radiation pneumonitis stratified by the predictive risk score model.

cumulative incidence of grade $\geq 2 \mathrm{RP}$ was $8.8 \%$ (5 patients), $13 \%$ ( 3 patients), and $84.6 \%$ (13 patients) when the PRS was $0,3-5$, and $6-11$, respectively $(\mathrm{P}<0.001)$ (Figure 2$)$. ROC curve analysis results revealed that combining age, $\mathrm{PE}$, and sILD was superior to PE or sILD alone (area under the curve $=0.814$ vs. 0.762 vs. 0.682$)$ (Figure 3).

\section{Discussion}

$\mathrm{RP}$ is an inevitable outcome that affects treatment effectiveness during and after lung cancer radiotherapy. Past studies have shown that RP incidence is correlated with dosimetric parameters and clinical factors (18). In the present study, $\mathrm{PO}_{2}$, age, sILD, $\mathrm{PE}$, and MLD were found to be the most significant predictors of grade $\geq 2$ RP. Our results are consistent with past reports on the use of dosimetric parameters (MLD, V20 and V5) to predict RP incidence. We performed a stratified analysis of various LA-NSCLC subtypes and found the results from SCC patients to be consistent with overall findings. However, few significant results were detected in ADE patients. Our analysis also indicated that PE, sILD, and age were correlated with RP incidence in SCC patients, and that patients with age $>68$ years, $\mathrm{PE}$ grade $>1$, and sILD grade $\geq 1$ on baseline CT may be more prone to RP. Our data showed that a model combining sILD, age, and PE accurately predicts RP. To our knowledge, the present study is the first to combine these factors.

We previously reported $\mathrm{PE}$ as an effective RP predictor in SCC patients, which is consistent with other studies (19). This could be because PE, a COPD subtype, can increase the possibility of DNA damage, causing RP. As known, 


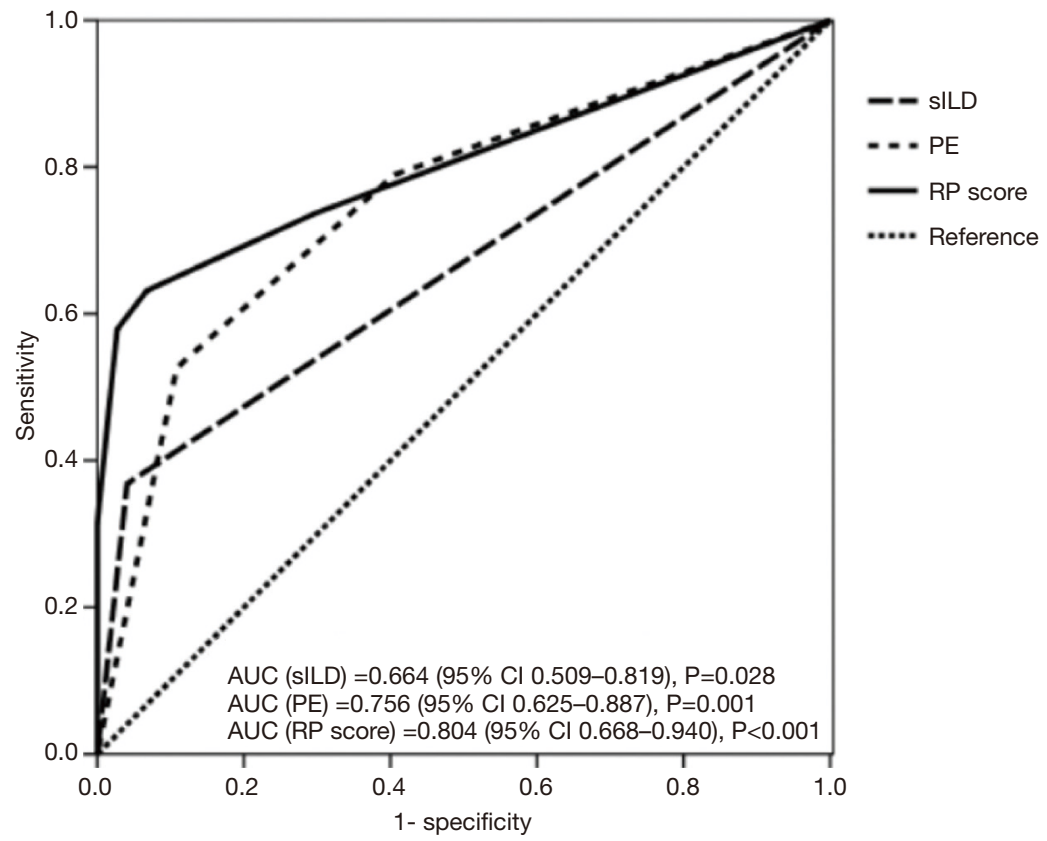

Figure 3 Receiving-operating characteristic curve based on the sensitivity and specificity of pulmonary emphysema (PE), subclinical interstitial lung disease (sILD) alone, or age, PE, and sILD score combined. AUC, area under the curve; RP, radiation pneumonitis.

SCC is a major type of lung cancer and can block the main trachea, reducing lung function, which may generate reactive oxygen species. However, some studies have indicated that PE is related to $\mathrm{RP}(20,21)$. A novel finding in the present study as that sILD, a pre-existing interstitial change, may increase the risk of severe RP (grade $\geq 2$ ) in LA-NSCLC patients. To the best of our knowledge, few studies have evaluated the relationship between thoracic radiotherapy and sILD in patients. Ueki et al. found that pre-existing ILD was a significant risk factor for grade $\geq 2 \mathrm{RP}$ (22). Okubo et al. found that, before SBRT, sILD is associated with grades $2-5 \mathrm{RP}$, which occurred in $45 \%(5 / 11)$ of their cases (23). Sanuki et al. reported that $3-26 \%$ of patients with pre-existing interstitial changes before radiotherapy developed severe RP (24). These reports suggest that sILD may increase the risk of severe RP and are consistent with our findings. However, thoracic radiotherapy may cause lymphocyte-mediated hypersensitivity reactions, which might exacerbate acute sILD (25). Therefore, accurately targeting the radiotherapy area and an accurate evaluation of subclinical ILD prior to radiotherapy are essential.

Advanced age is frequently associated with RP. Dang et al. conducted a multivariate analysis of 369 patients aged $>65$ years and found that more advanced age was a risk factor for grades 2 and $3 \mathrm{RP}$, which was consistent with our conclusion (26). This may due to the poor physical condition of older patients and increased comorbidities.

By adding beta coefficients for each significant variable, we developed a scoring system that predicts RP. Many predictive models for RP after lung cancer radiotherapy have been built in previous reports. Wang et al. found that combining interleukin-8, transforming growth factor (TGF)- $\beta 1$, and MLD into a RP prediction model improved its predictive ability relative to MLD alone (27). Krafft et al. found that the addition of CT radiomics extracted from the total lung significantly improved the model's capacity to predict RP (28). Tucker et al. showed that single nucleotide polymorphisms in the genes encoding transforming growth factor- $\beta$ (TGF- $\beta$ ), tumor necrosis factor- $\alpha$ (TNF $\alpha$ ), Vascular endothelial growth factor (VEGF), X-ray cross complementing 1 (XRCC1), X-ray cross complementing 3 (XRCC3), apyrimidinic endodeoxyribonuclease 1 (APEX1), and Methionine Synthase Reductase (MTRR) may significantly improve the predictive capacity of normal tissue complication probability (NTCP) models (29). Satoh et al. revealed that $\mathrm{V} 20 \geq 26 \%$, VS5 $\leq 1,500 \mathrm{cc}$, age $>68$ years, and pulmonary fibrosis could predict severe RP in LA-NSCLC patients treated with CCRT (17). In the present study, we combined sILD, PE, and age to develop an efficient and 
simple calculation method. This is the first report showing that age, sILD, PE, and the occurrence of those factors strongly correlate with severe RP in SCC patients. sILD is a novel risk factor for fatal RP. Consequently, older patients with higher PE and sILD grades should be closely monitored after radiotherapy.

The present study has some limitations. As it was retrospective study, selection bias was possible. Additionally, our model lacked dosimetric parameters and various biological markers, which are known to predict RP. Furthermore, our risk score has not been validated using an independent dataset. To overcome these limitations, larger studies are needed.

\section{Conclusions}

In the present retrospective study, age, PE, and sILD at baseline CT were independently and significantly associated with RP incidence in LA-NSCLC patients with SCC. By combining these three factors, an efficient and simple prediction model, which may be superior to single-factor models, was developed.

\section{Acknowledgments}

Funding: The work was funded by the Key Program of Science and Technology of Guangxi, China (Grant No. AB20159024) and General projects of Guangxi Natural Science Foundation (Grant No. 2018GXNSFAA281057).

\section{Footnote}

Reporting Checklist: The authors have completed the STARD reporting checklist. Available at http://dx.doi.org/10.21037/ apm-21-459

Data Sharing Statement: Available at http://dx.doi. org/10.21037/apm-21-459

Conflicts of Interest: All authors have completed the ICMJE uniform disclosure form (available at http://dx.doi. org/10.21037/apm-21-459). The authors have no conflicts of interest to declare.

Ethical Statement: The authors are accountable for all aspects of the work in ensuring that questions related to the accuracy or integrity of any part of the work are appropriately investigated and resolved. All procedures performed in this study involving human participants were in accordance with the Declaration of Helsinki (as revised in 2013). The present study was approved by the ethical review committee of Shanghai Pulmonary Hospital, Tongji University School of Medicine, China. Individual consent for this retrospective analysis was waived.

Open Access Statement: This is an Open Access article distributed in accordance with the Creative Commons Attribution-NonCommercial-NoDerivs 4.0 International License (CC BY-NC-ND 4.0), which permits the noncommercial replication and distribution of the article with the strict proviso that no changes or edits are made and the original work is properly cited (including links to both the formal publication through the relevant DOI and the license). See: https://creativecommons.org/licenses/by-nc-nd/4.0/.

\section{References}

1. Siegel RL, Miller KD, Jemal A. Cancer statistics, 2020. CA Cancer J Clin 2020;70:7-30.

2. Govindan R, Page N, Morgensztern D, et al. Changing epidemiology of small-cell lung cancer in the United States over the last 30 years: analysis of the surveillance, epidemiologic, and end results database. J Clin Oncol 2006;24:4539-44.

3. Sasaki T, Seto T, Yamanaka T, et al. A randomised phase II trial of S-1 plus cisplatin versus vinorelbine plus cisplatin with concurrent thoracic radiotherapy for unresectable, locally advanced non-small cell lung cancer: WJOG5008L. Br J Cancer 2018;119:675-82.

4. Rades D, Glatzel E, Werner EM, et al. Prevalence and Characteristics of Symptomatic Pneumonitis After Radiotherapy of Patients With Locally Advanced Lung Cancer. Anticancer Res 2019;39:6909-13.

5. Zhang T, Bi N, Zhou Z, et al. The impact of age on the survival outcomes and risk of radiation pneumonitis in patients with unresectable locally advanced non-small cell lung cancer receiving chemoradiotherapy. J Thorac Dis 2020;12:4347-56.

6. Jackson A, Marks LB, Bentzen SM, et al. The lessons of QUANTEC: recommendations for reporting and gathering data on dose-volume dependencies of treatment outcome. Int J Radiat Oncol Biol Phys 2010;76:S155-60.

7. Vogelius IR, Bentzen SM. A literature-based meta-analysis of clinical risk factors for development of radiation induced pneumonitis. Acta Oncol 2012;51:975-83.

8. Kong FM, Wang S. Nondosimetric risk factors for 
radiation-induced lung toxicity. Semin Radiat Oncol 2015;25:100-9.

9. Graham MV, Purdy JA, Emami B, et al. Clinical dosevolume histogram analysis for pneumonitis after $3 \mathrm{D}$ treatment for non-small cell lung cancer (NSCLC). Int J Radiat Oncol Biol Phys 1999;45:323-9.

10. Zhou Z, Song X, Wu A, et al. Pulmonary emphysema is a risk factor for radiation pneumonitis in NSCLC patients with squamous cell carcinoma after thoracic radiation therapy. Sci Rep 2017;7:2748.

11. Li F, Zhou Z, Wu A, et al. Preexisting radiological interstitial lung abnormalities are a risk factor for severe radiation pneumonitis in patients with small-cell lung cancer after thoracic radiation therapy. Radiat Oncol 2018;13:82.

12. Doyle TJ, Hunninghake GM, Rosas IO. Subclinical interstitial lung disease: why you should care. Am J Respir Crit Care Med 2012;185:1147-53.

13. Li Y, Liang S, Zhou Z, et al. Risk Factor Study of Radiation Pneumonitis Focused on Subclinical Interstitial Lung Disease in Definitive Radiation Therapy for NonSmall Cell Lung Cancer: A Single-Institution Experience. International journal of radiation oncology, biology, physics 2016;96:S131.

14. Liu H, Zhang X, Vinogradskiy YY, et al. Predicting radiation pneumonitis after stereotactic ablative radiation therapy in patients previously treated with conventional thoracic radiation therapy. Int J Radiat Oncol Biol Phys 2012;84:1017-23.

15. Tsujino K, Hashimoto T, Shimada T, et al. Combined analysis of V20, VS5, pulmonary fibrosis score on baseline computed tomography, and patient age improves prediction of severe radiation pneumonitis after concurrent chemoradiotherapy for locally advanced non-small-cell lung cancer. J Thorac Oncol 2014;9:983-90.

16. Washko GR, Hunninghake GM, Fernandez IE, et al. Lung volumes and emphysema in smokers with interstitial lung abnormalities. N Engl J Med 2011;364:897-906.

17. Satoh K, Kobayashi T, Misao T, et al. CT assessment of subtypes of pulmonary emphysema in smokers. Chest 2001;120:725-9.

18. Giuranno L, Ient J, De Ruysscher D, et al. RadiationInduced Lung Injury (RILI). Front Oncol 2019;9:877.

19. Kharofa J, Gore E. Symptomatic radiation pneumonitis in elderly patients receiving thoracic irradiation. Clin Lung Cancer 2013;14:283-7.
20. Kimura T, Togami T, Takashima H, et al. Radiation pneumonitis in patients with lung and mediastinal tumours: a retrospective study of risk factors focused on pulmonary emphysema. Br J Radiol 2012;85:135-41.

21. Ishijima $M$, Nakayama $H$, Itonaga $T$, et al. Patients with severe emphysema have a low risk of radiation pneumonitis following stereotactic body radiotherapy. Br J Radiol 2015;88:20140596.

22. Ueki N, Matsuo Y, Togashi Y, et al. Impact of pretreatment interstitial lung disease on radiation pneumonitis and survival after stereotactic body radiation therapy for lung cancer. J Thorac Oncol 2015;10:116-25.

23. Okubo M, Itonaga T, Saito T, et al. Predicting risk factors for radiation pneumonitis after stereotactic body radiation therapy for primary or metastatic lung tumours. Br J Radiol 2017;90:20160508.

24. Sanuki N, Ono A, Komatsu E, et al. Association of computed tomography-detected pulmonary interstitial changes with severe radiation pneumonitis for patients treated with thoracic radiotherapy. J Radiat Res 2012;53:110-6.

25. Roberts CM, Foulcher E, Zaunders JJ, et al. Radiation pneumonitis: a possible lymphocyte-mediated hypersensitivity reaction. Ann Intern Med 1993;118:696-700.

26. Dang J, Li G, Zang S, et al. Risk and predictors for early radiation pneumonitis in patients with stage III non-small cell lung cancer treated with concurrent or sequential chemoradiotherapy. Radiat Oncol 2014;9:172.

27. Wang S, Campbell J, Stenmark MH, et al. Plasma Levels of IL-8 and TGF- $\beta 1$ Predict Radiation-Induced Lung Toxicity in Non-Small Cell Lung Cancer: A Validation Study. Int J Radiat Oncol Biol Phys 2017;98:615-21.

28. Krafft SP, Rao A, Stingo F, et al. The utility of quantitative CT radiomics features for improved prediction of radiation pneumonitis. Med Phys 2018;45:5317-24.

29. Tucker SL, Li M, Xu T, et al. Incorporating singlenucleotide polymorphisms into the Lyman model to improve prediction of radiation pneumonitis. Int $\mathrm{J}$ Radiat Oncol Biol Phys 2013;85:251-7.

Cite this article as: $\mathrm{Wu} \mathrm{A}$, Zhou Z, Song Y, Liang S, Li F. Application of a radiation pneumonitis prediction model in patients with locally advanced lung squamous cell cancer. Ann Palliat Med 2021;10(4):4409-4417. doi: 10.21037/apm-21-459 